\title{
In Vivo Preliminary Study on Bone Neoformation Behavior of Three Types of Calcium Phosphate Bioceramics
}

\author{
Nelson H. A. Camargo'1, Enori Gemelli1, Aury N. de Moraes², Bruna D. da Costa², \\ Nilson Oleskovicz², Ademar L. Dallabrida², Doughlas Regalin², \\ Marcos Paulo Antunes de Lima² \\ ${ }^{1}$ Department Mechanical Engineering, Santa Catarina State University, Joinville, Brazil \\ ${ }^{2}$ Department of Veterinary Medicine, Santa Catarina State University, Lagres, Brazil \\ Email: dem2nhac@joinville.udesc.br, bruna ditzel@yahoo.com.br, a2anm@cav.udesc.br
}

Received December 2013

\begin{abstract}
Calcium phosphate microporous bioceramics and biphasic compositions of hydroxyapatite and $\beta$-calcium phosphate, in the form of microporous granular biomaterials, are research topics and present themselves as potential orthopedic and biomedical applications in rebuilding and repairing maxillofacial bones and tooth structure. This is associated with the characteristics of microstructure, biocompatibility, bioactivity and bone conductivity properties which these materials offer when applied in vivo or in a simulation environment. This study aimed to assess the behavior of bone neoformation of three types of calcium phosphate biomaterials in in vivo tests with sheep within 60 and 90 days, with the help of a scanning electron microscope. The biomaterials used were provided by the Group of Biomaterials at the Santa Catarina State University. The in vivo tests were carried out by generating, on sheep, tibial bone defects, three of which were filled with biomaterial (one different biomaterial for each bone defect generated), whilst the fourth received a bone fragment obtained during the generation of the defect in question, to serve as a control group. The scanning electron microscopy (SEM) technique was used for carrying out the preliminary characterization studies so as to observe new bone formation and osseointegration. The X-ray diffractometry (XRD) served as a support for the characterization of crystalline phases. The results obtained are encouraging and show that the biomaterials presented good performance in the process of bone formation, biomaterial osseointegration by a new tissue and bone mineralization.
\end{abstract}

\section{Keywords}

Bioceramics; Microstructure; Bone Neoformation; Characterization

\section{Introduction}

The calcium phosphate bioceramics and hydroxyapatite/b-calcium phosphate compositions are biocompatible

How to cite this paper: Camargo, N.H.A., et al. (2014) In Vivo Preliminary Study on Bone Neoformation Behavior of Three Types of Calcium Phosphate Bioceramics. Journal of Biosciences and Medicines, 2, 36-42. 
biomaterials which feature crystallographic similarity with the apatite in teeth and bone tissues, and play a main role in osteoinduction and osseointegration when applied in vivo [1] [2].

The calcium phosphate bioceramics in microporous blocks or granular form stand out in researches as biomaterials for repairing defects in bone tissues, as well as for reconstructing them, which has already been observed by different authors who carried out in vitro and in vivo tests, observing the good performance of these bioceramics on bone tissue neoformation [3] [4]. This is due to the fact that these biomaterials feature bioactivity, solubility, wettability and interconnected microporous microstructures, high micropore and grain surface area [5] [6]. These features are promising for adhesion of osteoblastic cells to the surface of granules through intergranular macropores. These microporous microstructures also favor the extension of osteoblastic cells to the interior of the granular biomaterial's interconnected microporosity, providing better conditions for bone tissue mineralization [5] [6]. These bioceramics are generally manufactured with an architecture composed of macro and microporosity, which has generated favorable results to the mechanism of cell adhesion and proliferation, thus contributing to the mechanisms of osteoinduction, repair and reconstruction of bone tissue [7]-[9].

The biomaterials used in this study were obtained with the wet chemical synthesis method, through the dissolution/precipitation involving liquid and solid phases of $\mathrm{CaO}$ and phosphoric acid solution, necessary for the formation of the molar $\mathrm{Ca} / \mathrm{P}$ compositions wanted. Three compositions of microporous granular biomaterials were used as follows: hydroxyapatite, b-calcium phosphate, and a 60\%HA/40\%b-TCP biphasic composition.

The in vivo tests were carried out by producing, on sheep, tibial diaphyseal bone defects, which were then filled by the respective biomaterials used in this study. One of the defects was filled with bone tissue to serve as a control. The results presented refer to studies of morphological and physical characterization of the biomaterials used in the in vivo tests. The characterization was executed with the scanning electron microscope (SEM) and X-ray diffractometry (XRD) techniques. The purpose of this study was to validate the biopsy preparation methodology for carrying out studies of characterization through scanning electron microscopy. The results obtained showed that the methodology used for biopsy preparation and the scanning electron microscopy technique allow assessment of these biomaterials' potential for osseointegration and bone tissue neoformation.

\section{Experimental Method}

In this study, three compositions of microporous granular calcium phosphate biomaterials were used: hydroxyapatite (HA), b-calcium phosphate (b-TCP) and biphasic 60\%HA/40\%b-TCP (HA/b-TCP). They were elaborated in the form of microporous granular biomaterials with sizes of $200 \mathrm{~mm}<\mathrm{d}<500 \mathrm{~mm}$.

Before the execution of in vivo tests, the project was first submitted to evaluation and approval by the Comitê de Ética and Bem-Estar Animal-CETEA) at the Santa Catarina State University, under the protocol number 1.29.12, approved for conducting in vivo tests with sheep. The microporous granular biomaterials were carefully sterilized at $280^{\circ} \mathrm{C} / 2 \mathrm{~h}$, packed, and subsequently used for in vivo tests with applications in sheep tibia.

The microstructural and morphological characterization studies were carried out with the help of a ZEISS DSM 940A scanning electron microscope, through the secondary electrons (SE) and backscattered electrons (BSE) system. These studies were carried out based on the microporous granular biomaterials and the biopsies taken from the in vivo tests with sheep, within 60 and 90 days. To facilitate the work of characterization through the scanning electron microscopy technique, fragments of biopsies were prepared with an aluminum alloy disc-shaped sample holder with smooth surface. A double sided carbon ribbon was attached to the disc's smooth surface, to which the biopsy fragment was carefully fixed. In order to avoid charging effects inside the microscope chamber, all biopsy fragments were submitted to a sputtering metallization process, with the deposition of a gold film on the surface of the biopsy fragment. This deposition was performed through a Bal-Tec Sputter Coater SCD 050 metallizer. The ion deposition parameters were established as follows: $25^{\circ} \mathrm{C}$ temperature inside the metallization chamber, $40 \mathrm{~mA}$ current, $2 \mathrm{kV}$ voltage, and 140 second deposition time, supplying a $30 \mathrm{~nm}$ gold film on the surface of the biopsy fragment.

The X-ray diffractometry (XRD) served as a support for the crystallographic characterization of microporous granular biomaterials. In these studies, a SHIMADZU LabX XRD-6000 X-ray diffractometer with a tube of copper anticathode was used. The X-ray diffractograms were obtained through the application of a $40 \mathrm{kV}$ voltage and $30 \mathrm{~mA}$ current intensity, with scanning angular range of $5^{\circ}$ to $80^{\circ}$ and a goniometer speed of $2^{\circ} / \mathrm{min}$ at 2q.

The biomaterials hydroxyapatite (HA), $\beta$-tricalcium phosphate $(\beta$-TCP) and 60/40 HA/ $\beta$-TCP were implanted 
in 6mm-diameter circular defects generated in sheep tibial diaphysis, respecting the same position of defect application in all animals.

Eight 12-month-old non-purebred female sheep were used. They had an average weight of $28.5 \pm 7.4 \mathrm{Kg}$ and were demonstrably healthy through clinical, laboratory, and radiographic examination. The animals were provided with antiparasitary medication, a corn silage-based diet, and ad libitum sheep feed and water. The animals received preanesthesic medication based on morphine $(0.3 \mathrm{mg} / \mathrm{Kg})$ and detomidine $(10 \mathrm{mg} / \mathrm{Kg})$, and were induced with a combination of diazepam $(0.5 \mathrm{mg} / \mathrm{Kg})$ and ketamine $(5 \mathrm{mg} / \mathrm{Kg})$. Maintenance of anesthesia was obtained with isoflurane diluted in $100 \%$ oxygen. Subarachnoid anesthesia was performed with a combination of levobupivacaine without vasoconstrictor $(0.5 \mathrm{mg} / \mathrm{Kg})$ and ketamine $(1.25 \mathrm{mg} / \mathrm{Kg})$. Initially, to start the surgery, the right pelvic limb was aseptically prepared. The access procedure was performed through the medial facet of the tibia, where the skin was incised, the subcutaneous tissue was divulsioned and the periosteum was incised. Two right tibial diaphyseal bone defects were generated. The proximal defect was filled with hydroxyapatite (HA), whilst the distal defect was filled with $\beta$-tricalcium phosphate $(\beta$-TCP). The same procedure was performed in the left pelvic limb, whose proximal defect was filled with an HA/ $\beta$-TCP (60/40) biphasic composition. The distal defect was filled with a bone fragment removed during the generation of the defect itself, simulating an autologous graft, in order to act as a control group. After the end of the procedure, the animals were intravenously given $0.6 \mathrm{mg} / \mathrm{Kg}$ of meloxicam and $25 \mathrm{mg} / \mathrm{Kg}$ of dipyrone. They also received three applications of antibiotic ( $20 \mathrm{mg} / \mathrm{Kg}$ of tetracycline every 48 hours). The meloxicam was maintained for two more days postoperatively and, from the second day on, $0.4 \mathrm{mg} / \mathrm{Kg}$ doses were applied intramuscularly. Animals also received, for three days, intramuscular applications of dipyrone $(25 \mathrm{mg} / \mathrm{Kg})$ every eight hours, and tramadol (6 $\mathrm{mg} / \mathrm{Kg}$ ) also every eight hours, as an analgesic supply. No animal presented postoperative complications such as infection, implant rejection, fractures or suture dehiscence.

The biopsies were first dehydrated in 70\% ethyl alcohol for 48 hours and then dehydrated again in absolute ethanol $100 \%$ for 56 hours. Later, they were put in xylene for 2 hours to clean the samples. Posteriorly, they were immersed once again in absolute ethanol $100 \%$ for 24 hours to eliminate the xylene. The biopsies obtained were fractured in the region where the bone fill was performed and were then prepared for the characterization studies with scanning electron microscopy (SEM).

\section{Results and Discussions}

The results of morphological and microstructural characterization, obtained with the microporous granular materials, revealed in their micrographs the morphology of the granules, with irregular shapes and sizes between $200 \mathrm{~mm}<\mathrm{d}<500 \mathrm{~mm}$, as it can be observed in Figures 1(a), 1(b), and 1(c).

Another observation was the interconnected microporous microstructure of the granular biomaterials, as featured in the micrographs represented by Figures 2(a), 2(b), and 2(c). By relating the micrographs obtained from the microporous granular biomaterials' microstructure, it is possible to observe a finer grain morphology, which indicates that there is greater microporosity in the HA granular biomaterials and biphasic composition, if compared to the b-TCP granular biomaterial.

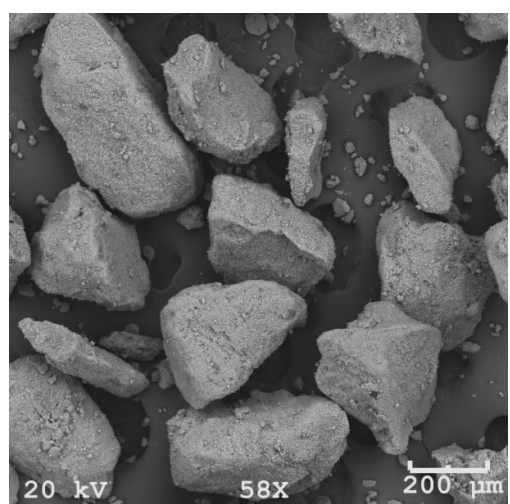

(a)

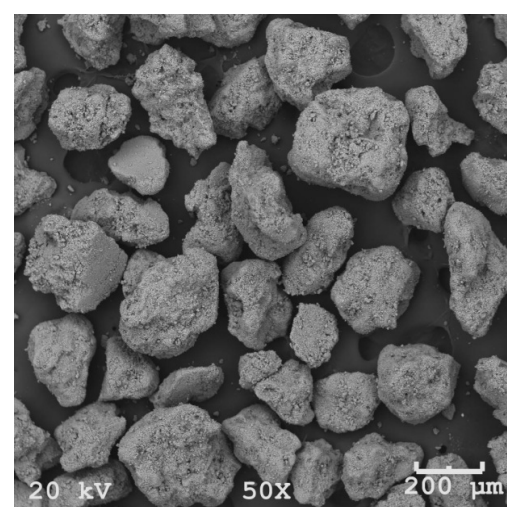

(b)

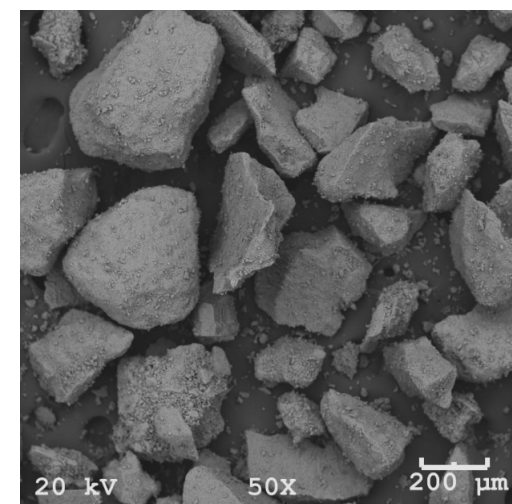

(c)

Figure 1. (a) Morphology of hydroxyapatite (HA) granular biomaterial; (b) b-TCP; (c) 60\%HA/40\%b-TCP biphasic granular biomaterial. 
The results obtained through X-ray diffractometry on the granular biomaterials clearly revealed, in their X-ray diphratograms, presence of the following phases: hydroxyapatite, b-calcium phosphate and hydroxyapatite/ b-calcium phosphate for biphasic composition, as featured in the X-ray diphratograms represented by Figures 3, 4 , and 5 .

The studies of characterization through scanning electron microscopy were carried out on the biopsy fragments from the in vivo tests within 60 and 90 days, regarding the biomaterials HA, b-TCP and $60 \% \mathrm{HA} / 40 \% \mathrm{~b}-$ TCP biphasic composition. These studies were carried out based on the microporous granular biomaterials and

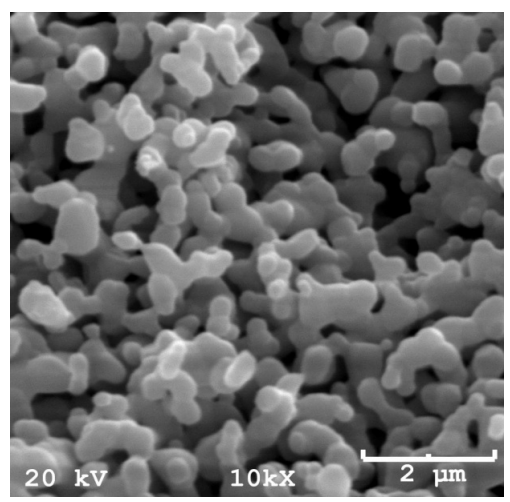

(a)

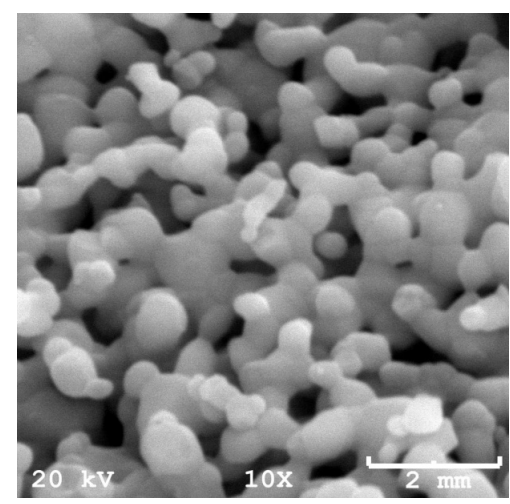

(b)

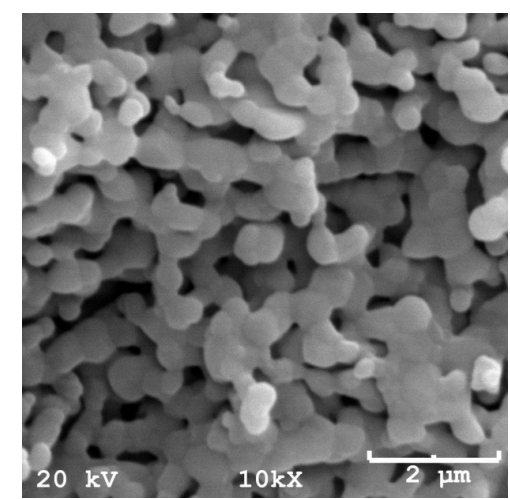

(c)

Figure 2. (a) Microstructure of HA granular biomaterial; (b) b-TCP granular biomaterial; (c) 60\%HA/40\%b-TCP biphasic granular biomaterial.

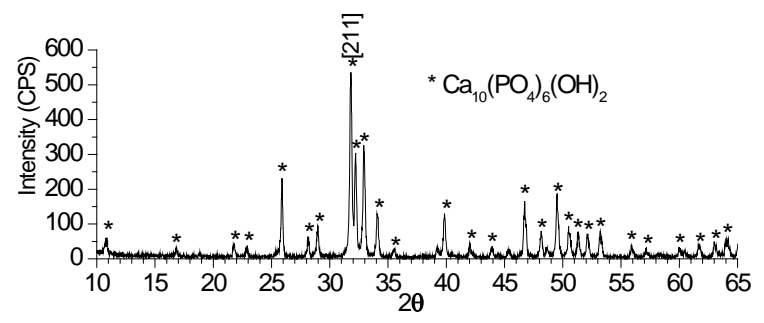

Figure 3. X-ray diphratogram obtained from HA granular biomaterial.

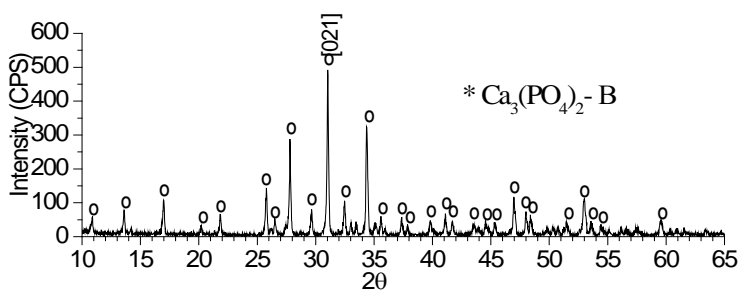

Figure 4. X-ray diphratogram obtained from b-TCP granular biomaterial.

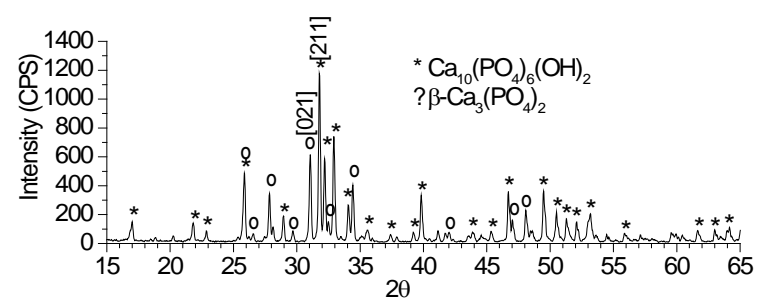

Figure 5. X-ray diphratogram obtained from HA/b-TCP biphasic granular biomaterial. 
the biopsies taken from the in vivo tests with sheep, within 60 and 90 days. The results revealed, in their micrographs, the presence of well formed bone tissue for the in vivo tests within 60 and 90 days, with better results in the tests within 90 days. (Figures 6(a), 6(b)) feature the micrographs obtained from the biopsies which contained the hydroxyapatite (HA) biomaterial for the test within 60 days. They clearly show good osseointegration and good quality of interface between biomaterial/neoformed bone tissue. Another observation was the internal microstructure of the remaining granular biomaterial. It can be clearly observed that there was a surface modification in the morphology of the initial biomaterial, if compared to Figure 2(a), a microstructure formed by consistent mass can be now observed, which indicates the presence of formed bone apatite, collagen and proteins (Figure 6(b)).

The results obtained from the in vivo tests within 90 days, with the hydroxyapatite biomaterial, revealed in their micrographs better quality of neoformed bone tissue, if compared to the results within 60 days, as illustrated in Figure 6(c). There was also good quality of biomaterial/bone tissue interface and modification in the internal microstructure of the remaining granular biomaterial, which was also observed within 60 days (Figure 6(d)).

The results obtained from the in vivo tests with the b-TCP microporous granular biomaterial, within 60 days, revealed in their micrographs good bone neoformation, osseointegration and biomaterial/new bone tissue interface (Figures 7(a) and 7(b)). Another observation was a modification in the microporous microstructure of the biomaterial, which had already been observed in the result obtained with HA. Results obtained from the test within 90 days revealed good performance by the b-TCP granular biomaterial on the process of osseointegration and bone neoformation. Another relevant point was the quality of interface between biomaterial, bone tissue formed and its mineralization (Figures 7(c), 7(d)).

The results obtained from the biopsies containing HA/b-TCP biphasic biomaterial, with the in vivo test within 60 days, revealed in their micrographs good performance of mineralization and bone formation (Figure 8(a)). Good osseointegration and quality of biomaterial/neoformed bone tissue interface were also observed (Figure $\mathbf{8 ( b ) )}$. After observing the results of bone neoformation between the biomaterials within 60 days, it can be said that the biphasic biomaterial presented superior results regarding bone neoformation. The results obtained within 90 days also revealed in their micrographs good bone formation and osseointegration of the biomaterial by the new bone tissue formed (Figures 8(c) and 8(d)).

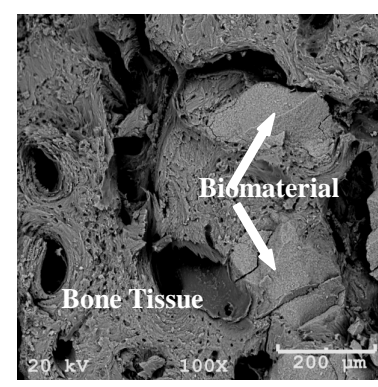

(a)

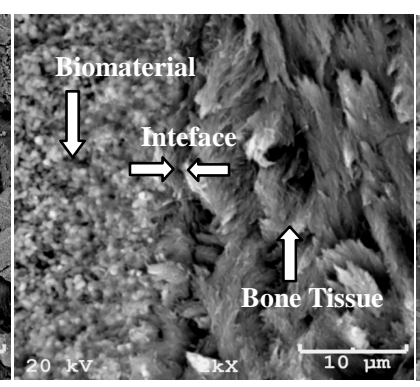

(b)

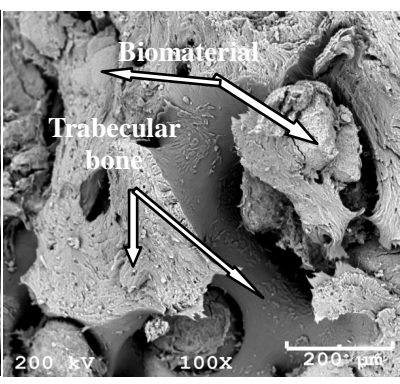

(c)

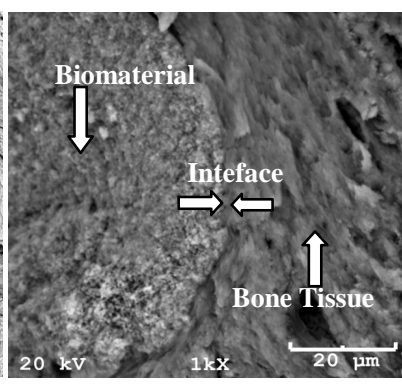

(d)

Figure 6. (a) HA biopsy, 60 days, osseointegration; (b) Quality of biomaterial/bone tissue interface; (c) HA biopsy, 90 days, well formed bone tissue; (d) Quality of biomaterial/bone tissue interface.

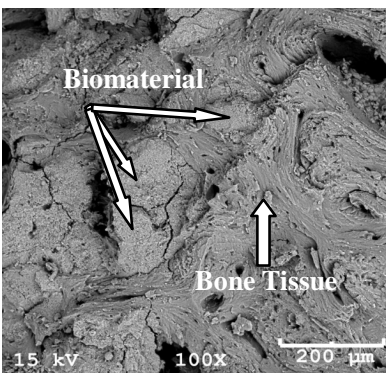

(a)

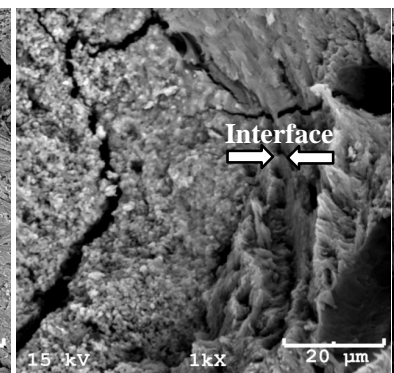

(b)

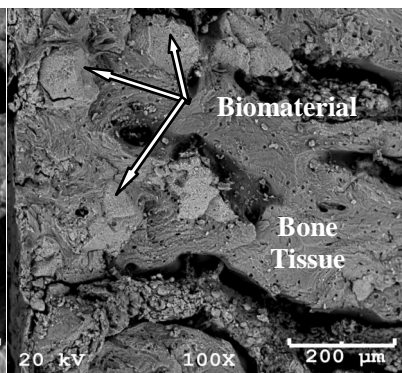

(c)

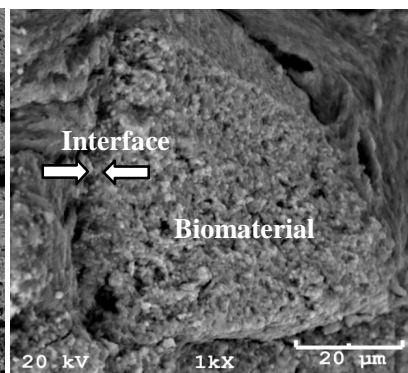

(d)

Figure 7. (a) b-TCP biopsy, 60 days, bone tissue and osseointegration; (b) Biomaterial/bone tissue interface; (c) b-TCP biopsy, 90 days, osseointegration; (d) Remaining biomaterial/bone tissue interface. 


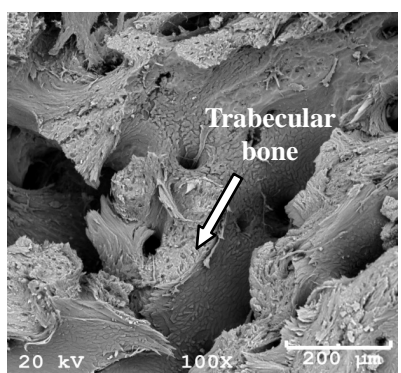

(a)

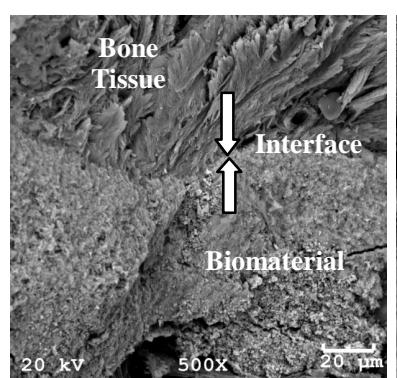

(b)

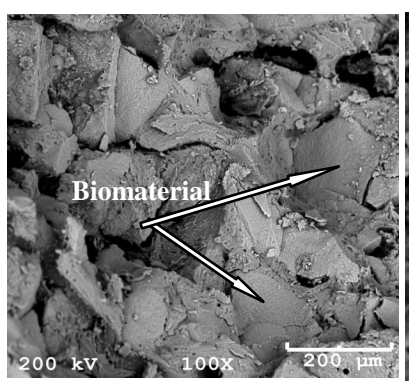

(c)

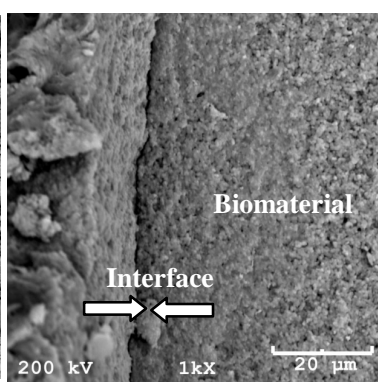

(d)

Figure 8. (a) HA/b-TCP biopsy, 60 days, trabecular bone tissue; (b) Osseointegration and interface; (c) HA/b-TCP biopsy, 90 days, osseointegration; (d) Biomaterial/bone tissue interface.

The results from the in vivo tests for the three types of biomaterial evidenced, through the studies with scanning electron microscopy, better results in bone neoformation within 90 days, which is explained by the permanence time of the biomaterial in the animal. Another finding was the presence of remaining biomaterial within both 60 and 90 days; however, there was presence of greater amount of remaining biomaterial in the results obtained within 60 days. The good quality of osseointegration and of interface between biomaterial and new bone tissue formed was observed in both times (60 and 90 days). Apparently, the best result of bone neoformation verified was with the biphasic biomaterials within 90 days. However, in general, it can be said that the three microporous granular biomaterials of HA, b-TCP and HA/b-TCP presented relevant results of bone neoformation within 60 and 90 days. This preliminary study of biopsy preparation and the scanning electron microscopy technique have allowed an assessment of quality in the neoformed bone tissue and the osseointegration of the biomaterial by the new bone tissue formed.

\section{Conclusion}

The morphological and microstructural characterization clearly showed that the biomaterials are formed by granules with various shapes and microporous microstructures formed by thin grains. These characteristics are promising to cell adhesion and proliferation on the surface of grains and micropores, as observed in the results obtained within 60 and 90 days. The results obtained through X-ray diffractometry evidenced in their diphratograms the respective phases of each biomaterial. The studies on the biopsies clearly showed a good performance by the biomaterials in the process of bone neoformation and osseointegration. It can be stated that, in this preliminary study, the biphasic biomaterial slightly stood out on bone neoformation and osseointegration. This could be associated to this biomaterial's microporosity and better solubility. The preliminary results are encouraging and demonstrate that the granular biomaterials are promising as bone substitutes. The method of biopsy preparation for the studies with scanning electron microscopy has proven to be effective and allowed to reveal the microstructure of the bone tissue formed and the morphology of the remaining biomaterial.

\section{References}

[1] Camargo, N.H.A., de Lima, S., Souza, J.C.P., de Aguiar, J.F., Gemelli, E., Meier, M.M., Silva, V.E. and Mittelstädt, F.G. (2009) Synthesis and Characterization of Nanostructured Ceramic Powders of Calcium Phosphate and Hydroxyapatite for Dental Applications. Key Engineering Materials, 398, 619-622. http://dx.doi.org/10.4028/www.scientific.net/KEM.396-398.619

[2] Yuan, H., Kurashina, K., de Bruijn, J.D., Li, Y. and de Groot, K. (1999) A Preliminary Study on Osteoinduction of Two Kinds Calcium Phosphate Ceramics. Biomaterials, 20, 1799-1806.

[3] Kiurashina, K., Kurita, H., Wu, Q., Ohtsuka, A. and Kobayashi H. (2002) Ectopic Osteogenesis with Biphasic Ceramics of Hydroxyapatite and Tricalcium Phosphate in Rabbits. Biomaterials, 23, 407-412.

[4] Ramay, H.R.R. and Zhang, M. (2004) Biphasic Calcium Phosphate Nanocomposite Porous Scaffolds for Load-Bearing Bone Tissue Engineering. Biomaterials, 25, 5171-5180.

[5] Tanaka, T., Kitasato, S., Chazono, M., Kumaggae, Y., Iida, T., Mitsuhashi, M., Kauta, A. and Marumo, K. (2012) Use of an Injectable Complex of Tricalcium Phosphate Granules, Hyaluronate, and Fibroblast Growth Factor-2 on Repair of Unstable Intertrochanteric Fractures. The Open Biomedical Engineering Journal, 6, 98-103. 
[6] Camargo, N.H.A., de Lima, S.A. and Gemelli, E. (2012) Synthesis and Characterization of Hydroxyapatite/TiO $2 \mathrm{n}$ Nanocomposites for Bone Tissue Regeneration. American Journal of Biomedical Engineering, 2, 41-47.

[7] Rosa, A.L., Beloti, M.M., Oliveira, P.T., Van Noort, R. (2002) Osseointegration and Osseoconductivity of Hydroxyapatite of Different Microporosite. Journal of Materials Science: Materials in Medicine, 13, 1071-1075.

[8] Dorozhkin, S.V. (2012) Biphasic, Triphasic and Multiphasic Calcium Phosphetes. Acta Biomaterialia, 8, 963-977.

[9] Ramay, H.R.R. and Zhang, M. (2004) Biphasic Calcium Phosphate Nanocomposite Porous Scaffolds for Load-Bearing Bone Tissue Engineering. Biomaterials, 25, 5171-5180. 